CRYSTALLOGRAPHIC COMMUNICATIONS

ISSN 2056-9890

Received 10 October 2018

Accepted 29 October 2018

Edited by M. Weil, Vienna University of Technology, Austria

Keywords: crystal structure; BEDT-TTF; $\mathrm{Cu}(\mathrm{NCS})_{2}$; superconductor; pseudo- $\kappa$ arrangement.

CCDC reference: 1876009

Supporting information: this article has supporting information at journals.iucr.org/e

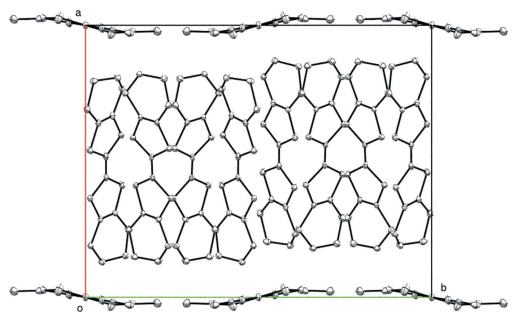

OPEN $\odot$ ACCESS

\section{A new compound in the BEDT-TTF family [BEDT-TTF = bis(ethylenedithio)tetrathiafulvalene] with a tetrathiocyanatocuprate(II) anion, $(\text { BEDT-TTF })_{4}\left[\mathrm{Cu}(\mathrm{NCS})_{4}\right]$}

Christophe Faulmann,* Benoît Cormary, Lydie Valade, Kane Jacob and Dominique de Caro

CNRS, LCC (Laboratoire de Chimie de Coordination), 205 route de Narbonne, BP44099, 31077 Toulouse Cedex 4, France, Université de Toulouse, UPS, INPT, 31077 Toulouse Cedex 4, France. *Correspondence e-mail: christophe.faulmann@|cc-toulouse.fr

A new phase combining BEDT-TTF and $\left[\mathrm{Cu}(\mathrm{NCS})_{4}\right]^{2-}$ as the counter-anion, namely bis[bis(ethylenedithio)tetrathiafulvalenium] tetrathiocyanatocuprate(II) bis[bis(ethylenedithio)tetrathiafulvalene], $\left(\mathrm{C}_{10} \mathrm{H}_{8} \mathrm{~S}_{8}\right)_{2}\left[\mathrm{Cu}(\mathrm{NCS})_{4}\right] \cdot-$ $2 \mathrm{C}_{10} \mathrm{H}_{8} \mathrm{~S}_{8}$ or $(\mathrm{BEDT}-\mathrm{TTF})_{4}\left[\mathrm{Cu}(\mathrm{NCS})_{4}\right]$ was obtained during a galvanostatic electrocrystallization process. As previously observed with BEDT-TTF-based compounds with oxalatometallate anions, the BEDT-TTF molecules in (BEDT$\mathrm{TTF})_{4}\left[\mathrm{Cu}(\mathrm{NCS})_{4}\right]$ exhibit the so-called pseudo- $\kappa$ arrangement, with two BEDTTTF molecules being positively charged and two electronically neutral. The bond lengths and angles in the two unique BEDT-TTF molecules differ slightly. The crystal structure consists of layers of BEDT-TTF molecules extending parallel to (001). The width of this layer corresponds to the length of the $a$ axis [16.9036 (17) A]. The BEDT-TTF layers are separated by layers of centrosymmetric square-planar $\left[\mathrm{Cu}(\mathrm{NCS})_{4}\right]^{2-}$ dianions.

\section{Chemical context}

For several years, we have been interested in synthesizing molecular (super)conductors as nanoparticles (Chtioui-Gay et al., 2016; Valade et al., 2016; de Caro, Jacob et al., 2013; de Caro, Souque et al., 2013; de Caro et al., 2014; Winter et al., 2015 ) in order to study the effects of size reduction on the properties of this kind of material. As there are numerous structuring agents such as ionic liquids based, for instance, on imidazolium cations (Fig. 1), and long alkyl chains in ammonium salts or neutral amines, it is possible to obtain nanoparticles of these materials, either by electrochemical oxidation or by chemical reaction. Recently, we have focused on BEDT-TTF-based compounds [BEDT-TTF is bis(ethylenedithio)tetrathiafulvalene]. The BEDT-TTF family is one of the most studied in the field of molecular superconductors because it exhibits the largest number of superconductors with $T_{\mathrm{c}}$ above $10 \mathrm{~K}$ (Ishiguro et al., 1998). During the planned electrosynthesis of (BEDT-TTF $)_{2}\left[\mathrm{Cu}(\mathrm{NCS})_{2}\right]$ as nanoparticles

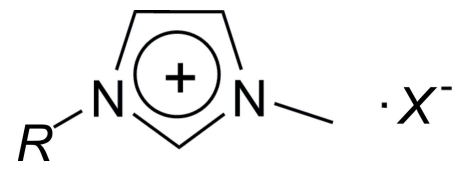

Figure 1

Examples of ionic liquids with the imidazolium fragment. For example, $R$ = butyl: $\mathrm{BMI} M^{+} ; R=$ ethyl: $\mathrm{EMI}^{+} ; X=\mathrm{PF}_{6}^{-}, \mathrm{BF}_{4}^{-}, \mathrm{SCN}^{-}$. 
from BEDT-TTF and $\mathrm{Cu}(\mathrm{SCN})$ in the presence of (EMIM)(SCN) (EMIM = 1-ethyl-3-methylimidazolium), a few crystals were formed as a minor product besides the desired powder as the main phase. A structure determination of these crystals revealed a new salt-like compound, based on the BEDT-TTF donor and the $\left[\mathrm{Cu}(\mathrm{NCS})_{4}\right]^{2-}$ dianion, namely pseudo- $\kappa$-(BEDT-TTF $)_{4}\left[\mathrm{Cu}(\mathrm{NCS})_{4}\right]$.<smiles>[Z]CSCC[C@H](C)O</smiles>

\section{Structural commentary}

The asymmetric unit of the title salt contains two well-ordered BEDT-TTF molecules and one $\mathrm{Cu}(\mathrm{NCS})_{2}$ entity with the $\mathrm{Cu}^{\mathrm{II}}$ cation lying on an inversion centre (Fig. 2). This results in the composition (BEDT-TTF $)_{4}\left[\mathrm{Cu}(\mathrm{NCS})_{4}\right]$, and thus is different from the well-known $\kappa$-phase (BEDT-TTF $)_{2}\left[\mathrm{Cu}(\mathrm{NCS})_{2}\right]$ (Hiramatsu et al., 2015; Schultz et al. 1991; Urayama et al., $1988)$ and also from (BEDT-TTF) $\left[\mathrm{Cu}_{2}(\mathrm{NCS})_{3}\right]$ (Geiser et al., 1988). One of the two BEDT-TTF molecules (central bond $\mathrm{C} 7-\mathrm{C} 8$ ) forms a dimer that is related through an inversion centre, whereas the other BEDT-TTF molecules (central bond $\mathrm{C} 17-\mathrm{C} 18)$ are farther away from each other. To our knowledge, this feature has not been observed within the (BEDT$\mathrm{TTF})\left[\mathrm{Cu}(\mathrm{NCS})_{x}\right]$ family, but it has been found in BEDT-TTF compounds with tris-(oxalato)metallate anions, such as $(\mathrm{BEDT}-\mathrm{TTF})_{4}\left[A M\left(\mathrm{C}_{2} \mathrm{O}_{4}\right)_{3}\right] \cdot$ solv. $\left(A=\mathrm{K}, \mathrm{NH}_{4}, \mathrm{H}_{3} \mathrm{O} ; M=\mathrm{Fe}\right.$, $\mathrm{Cr}, \mathrm{Co}, \mathrm{Ru}$; solv. = benzonitrile, 1,2-dichlorobenzene, bromobenzene) (Kurmoo et al., 1995; Martin et al., 2001; Prokhorova et al., 2011, 2013). The latter compounds are representatives of

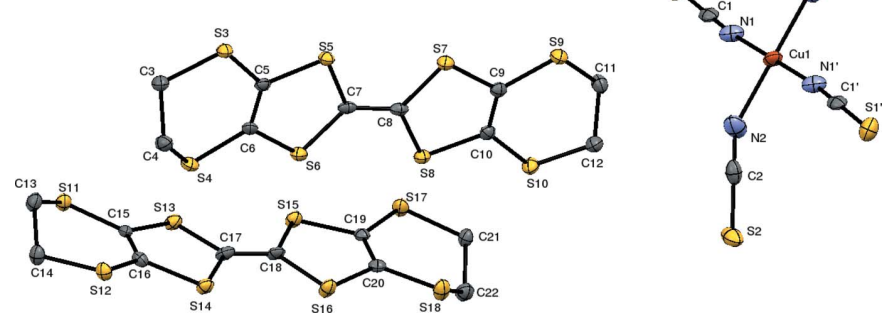

Figure 2

Molecular structure of (BEDT-TTF $)_{4}\left[\mathrm{Cu}(\mathrm{NCS})_{4}\right]$, showing the BEDTTTF molecule involved in the formation of a dimer (top), and the other BEDT-TTF molecule (bottom), as well as the centrosymmetric $\left[\mathrm{Cu}(\mathrm{NCS})_{4}\right]^{2-}$ dianion. Displacement ellipsoids are drawn at the $50 \%$ probability level. $\mathrm{H}$ atoms have been omitted for clarity. Primed atoms are generated by the symmetry operation $(-x, 1-y, 2-z)$.

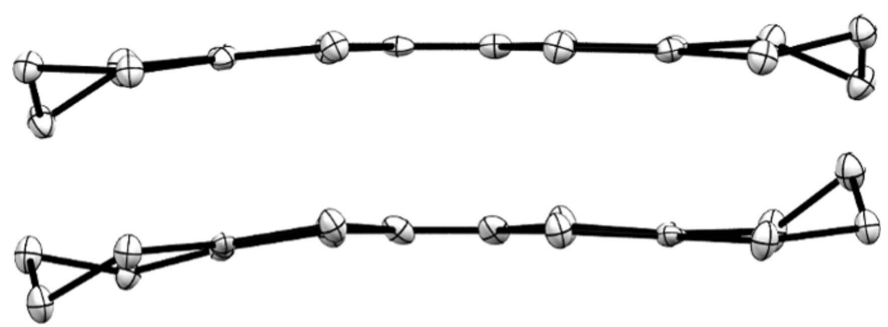

Figure 3

Side view of the BEDT-TTF molecules (top: in the dimer; bottom: the other molecule). Displacement ellipsoids are drawn at the $50 \%$ probability level.

the pseudo $\kappa$-phase where the two independent BEDT-TTF molecules show some slight structural differences. Similarly, the bond lengths within the central $\mathrm{C}_{2} \mathrm{~S}_{4}$ core in the BEDTTTF molecules of the title salt deviate by up to $0.035 \AA$. The bond lengths in the TTF core are indicative of the degree of charge in this family of BEDT-TTF compounds. According to Guionneau et al. (1997), this allows the charge $Q$ of the two BEDT-TTF molecules in the title salt to be calculated. Whereas each BEDT-TTF molecule in the dimer carries a charge of $+1(Q=0.83)$, the other BEDT-TTF molecule is neutral $(Q=0.18)$. Not only do the bond lengths of the BEDTTTF molecules in the title salt show some differences, but the overall shape of the molecules also differs. The BEDT-TTF molecule in the dimer deviates less from planarity [r.m.s. deviation of $0.0853 \AA$ neglecting the outer ethylene bridges, with the largest deviation being 0.1579 (19) $\AA$ for S5] than the other BEDT-TTF molecule [r.m.s. deviation of $0.1431 \AA$; highest deviation $=0.3273$ (12) $\AA$ for S12]. Moreover, the outer ethylene groups tend to be more eclipsed in the molecule of the dimer whereas they tend to be more staggered in the other molecule (Fig. 3). All these features are similar to those reported for the $(\mathrm{BEDT}-\mathrm{TTF})_{4}\left[A M\left(\mathrm{C}_{2} \mathrm{O}_{4}\right)_{3}\right] \cdot$ solv. family.

Contrary to what is observed in other BEDT-TTF compounds associated with $\left[\mathrm{Cu}(\mathrm{NCS})_{x}\right]$ anions that are present as polymeric $\left[\mathrm{Cu}(\mathrm{NCS})_{x}\right]$ entities, in the title compound discrete $\left[\mathrm{Cu}(\mathrm{NCS})_{4}\right]^{2-}$ units are observed. The $\mathrm{Cu}^{\mathrm{II}}$ cation (which lies on a centre of inversion) of the $\left[\mathrm{Cu}(\mathrm{NCS})_{4}\right]^{2-}$ dianion adopts an almost regular square-planar $\mathrm{CuN}_{4}$ environment, with $\mathrm{N}-\mathrm{Cu}-\mathrm{N}$ angles close to 90 and $180^{\circ}$. Intramolecular bond lengths in the anion are in agreement with other tetrathiocyanatocuprates(II) (Wang et al., 2008; Chekhlov, 2009). It should be noted that one $\mathrm{Cu}-\mathrm{NCS}$ fragment is more bent than the other one [angle $\mathrm{Cu}-\mathrm{N} 2-\mathrm{C} 2$ of $151.6(4)^{\circ}$ versus $175.2(3)^{\circ}$ for $\mathrm{Cu}-\mathrm{N} 1-\mathrm{C} 1$; Fig. 2].

\section{Supramolecular features}

The BEDT-TTF molecules in the dimer stack face-to-face. The interplanar distance within the dimer is 3.62 (3) $\AA$, considering the least-squares planes of the molecule except for the terminal ethylene groups. In addition, there are two pairs of short S..S contacts within the dimer $[\mathrm{S} 5 \cdots \mathrm{S} 8=3.461$ (1) and $\mathrm{S} 6 \cdots \mathrm{S} 7=3.515(1) \AA]$. Each dimer is surrounded by six 


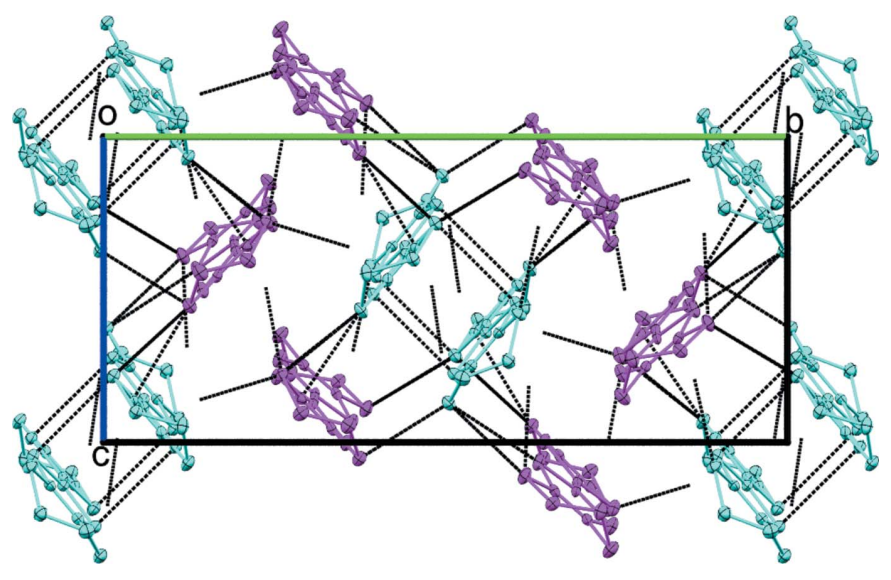

Figure 4

View along [100] of the packing of the molecular entities in the crystal structure of (BEDT-TTF $)_{4}\left[\mathrm{Cu}(\mathrm{NCS})_{4}\right]$. The dimer of BEDT-TTF molecules (turquoise) is surrounded by other BEDT-TTF molecules (purple). Black dotted lines represent short S...S and S...H contacts shorter than the sum of the van der Waals radii (for numerical details, see: Table 1).

adjacent BEDT-TTF molecules (Fig. 4). Four of them lie nearly perpendicular to the dimer [angle of $86.16(2)^{\circ}$ ] and are connected with the dimer through short S...S contacts (Table 1) and the two others are almost parallel to the dimer [angle of $7.09(4)^{\circ}$ ] with short S $\cdots H$ contacts (Table 1). This arrangement leads to layers of BEDT-TTF donors, extending parallel to (001). The layers have a width that corresponds to the length of the $a$ axis and are separated from each other by layers of $\left[\mathrm{Cu}(\mathrm{NCS})_{4}\right]^{2-}$ dianions (Fig. 5).

\section{Synthesis and crystallization}

(BEDT-TTF $)_{4}\left[\mathrm{Cu}(\mathrm{NCS})_{4}\right]$ was prepared by galvanostatic electrocrystallization in an $\mathrm{H}$-shaped cell, equipped with $\mathrm{Pt}$ electrodes and with a glass frit between the anodic and cathodic compartments. To the cathodic compartment were added $\operatorname{EMIM}(\mathrm{SCN})(\mathrm{EMIM}=1$-ethyl-3-methylimidazolium;

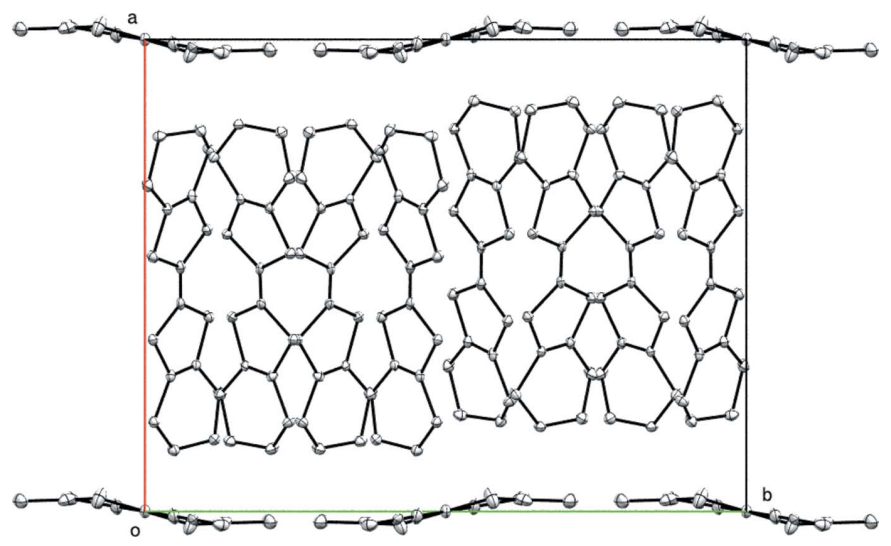

Figure 5

View of the structural arrangement of (BEDT-TTF $)_{4}\left[\mathrm{Cu}(\mathrm{NCS})_{4}\right]$, showing layers of BEDT-TTF molecules parallel to (001) separated by layers of $\left[\mathrm{Cu}(\mathrm{NCS})_{4}\right]^{2-}$ dianions.

Table 1

Table of contacts $(\AA)$ shorter than the sum of the van der Waals radii.

\begin{tabular}{|c|c|c|}
\hline Atom $1 \cdots$ atom 2 & Length & Symmetry operation on atom 2 \\
\hline $\mathrm{S} 5 \cdots \mathrm{S} 8$ & $3.461(1)$ & $1-x, 1-y, 1-z$ \\
\hline 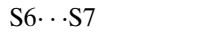 & $3.515(1)$ & $1-x, 1-y, 1-z$ \\
\hline $\mathrm{S} 4 \cdot \cdots \mathrm{S} 12$ & $3.463(1)$ & $x, y, z$ \\
\hline S4 $\cdots$ S14 & $3.586(1)$ & $x, y, z$ \\
\hline $\mathrm{S} 10 \cdots \mathrm{H} 21 B$ & 2.76 & $x, y, z$ \\
\hline $\mathrm{H} 12 A \cdots \mathrm{S} 17$ & 2.81 & $x, y, 1+z$ \\
\hline 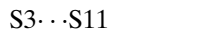 & $3.511(1)$ & $x, y, 1+z$ \\
\hline $\mathrm{S} 5 \cdots \mathrm{S} 11$ & $3.508(1)$ & $x, y, 1+z$ \\
\hline S9 $\cdots$ S15 & $3.571(1)$ & $x, y, 1+z$ \\
\hline S9..S17 & $3.574(1)$ & $x, y, 1+z$ \\
\hline S3 $\cdots$ S17 & $3.470(1)$ & $1-x, 1-y, 1-z$ \\
\hline $\mathrm{S} 5 \cdots \mathrm{S} 17$ & $3.533(1)$ & $1-x, 1-y, 1-z$ \\
\hline S9 $\cdots$ S11 & $3.522(1)$ & $1-x, 1-y, 1-z$ \\
\hline $\mathrm{H} 4 B \cdots \mathrm{S} 12$ & 2.74 & $x, \frac{3}{2}-y, \frac{1}{2}+z$ \\
\hline $\mathrm{S} 12 \cdots \mathrm{H} 14 A$ & 2.73 & $x, \frac{3}{2}-y, \frac{1}{2}+z$ \\
\hline
\end{tabular}

$60 \mu \mathrm{l}, 0.4 \mathrm{mmol})$ and freshly distilled 1,1,2-TCE (TCE = trichlorethylene; $10 \mathrm{ml}) . \mathrm{Cu}(\mathrm{NCS})(8 \mathrm{mg}, 0.07 \mathrm{mmol})$ and $\operatorname{EMIM}(\mathrm{SCN})(60 \mu \mathrm{L}, 0.4 \mathrm{mmoL})$ were added to the anodic compartment, which was immediately filled with BEDT-TTF (30 mg, $0.08 \mathrm{mmol}$ ), previously dissolved in $10 \mathrm{ml}$ of 1,1,2-TCE at $343 \mathrm{~K}$. The current was set at $100 \mu \mathrm{A}$ (current density of $318 \mu \mathrm{A} \mathrm{cm}{ }^{-2}$ ). After $12 \mathrm{~h}$, a black powder corresponding to the desired compound (BEDT-TTF $)_{2}\left[\mathrm{Cu}(\mathrm{NCS})_{2}\right]$ was harvested by filtration, together with some crystals of (BEDT$\mathrm{TTF})_{4}\left[\mathrm{Cu}(\mathrm{NCS})_{4}\right]$, which were washed with $1,1,2$-TCE and dried under vacuum.

Table 2

Experimental details.

Crystal data

Chemical formula

$M_{\mathrm{r}}$

Crystal system, space group

Temperature (K)

$a, b, c(\AA)$

$\beta\left({ }^{\circ}\right)$

$V\left(\AA^{3}\right)$

$Z$

Radiation type

$\mu\left(\mathrm{mm}^{-1}\right)$

Crystal size (mm)

Data collection

Diffractometer

Absorption correction

$T_{\min }, T_{\max }$

No. of measured, independent and observed $[I>2 \sigma(I)]$ reflections

$R_{\text {int }}$

$\theta_{\max }\left({ }^{\circ}\right)$

$(\sin \theta / \lambda)_{\max }\left(\AA^{-1}\right)$

Refinement

$R\left[F^{2}>2 \sigma\left(F^{2}\right)\right], w R\left(F^{2}\right), S$

No. of reflections

No. of parameters

$\mathrm{H}$-atom treatment

$\Delta \rho_{\max }, \Delta \rho_{\min }\left(\mathrm{e} \AA^{-3}\right)$

Computer programs: APEX2 and SAINT (Bruker, 2012), SHELXS97 (Sheldrick, 2008), SHELXL2018 (Sheldrick, 2015), Mercury (Macrae et al., 2006) and WinGX (Farrugia, 2012).
$\left(\mathrm{C}_{10} \mathrm{H}_{8} \mathrm{~S}_{8}\right)_{2}\left[\mathrm{Cu}(C N S)_{4}\right] \cdot 2 \mathrm{C}_{10} \mathrm{H}_{8} \mathrm{~S}_{8}$ 1834.43

Monoclinic, $P 2_{1} / c$

100

16.9036 (17), 21.004 (2), 9.6205 (9)

$103.071(3)$

$3327.1(6)$

2

Mo $K \alpha$

1.50

$0.18 \times 0.16 \times 0.02$

Bruker Kappa APEXII Quazar CCD

Multi-scan (SADABS; Bruker, 2012)

$0.652,0.745$

$48335,5028,3816$

0.070

23.7

0.566

$0.032,0.078,1.02$

5028

385

$\mathrm{H}$-atom parameters constrained $0.38,-0.39$ 


\section{Refinement}

Crystal data, data collection and structure refinement details are summarized in Table 2. The crystal diffracted rather weakly $\left(2 \theta_{\max }=47.42^{\circ}\right)$. The hydrogen atoms of the ethylene bridges were placed in idealized positions and were refined with $\mathrm{C}-\mathrm{H}=0.99 \AA$ and with $U_{\text {iso }}(\mathrm{H})=1.2 U_{\text {eq }}(\mathrm{C})$. Reflections (100) and (110) were obstructed by the beam stop and thus were excluded from the refinement.

\section{Acknowledgements}

Sonia Mallet-Ladeira is acknowledged for her help and discussions related with X-ray diffraction.

\section{References}

Bruker (2012). APEX2, SAINT and SADABS. Bruker AXS Inc., Madison, Wisconsin, USA.

Caro, D. de, Faulmann, C., Valade, L., Jacob, K., Chtioui, I., Foulal, S., de Caro, P., Bergez-Lacoste, M., Fraxedas, J., Ballesteros, B., Brooks, J. S., Steven, E. \& Winter, L. E. (2014). Eur. J. Inorg. Chem. pp. 4010-4016.

Caro, D. de, Jacob, K., Faulmann, C. \& Valade, L. (2013). C. R. Chim. 16, 629-633.

Caro, D. de, Souque, M., Faulmann, C., Coppel, Y., Valade, L., Fraxedas, J., Vendier, O. \& Courtade, F. (2013). Langmuir, 29, 8983-8988.

Chekhlov, A. N. (2009). Russ. J. Gen. Chem. 79, 744-748.

Chtioui-Gay, I., Faulmann, C., de Caro, D., Jacob, K., Valade, L., de Caro, P., Fraxedas, J., Ballesteros, B., Steven, E., Choi, E. S., Lee, M., Benjamin, S. M., Yvenou, E., Simonato, J. P. \& Carella, A. (2016). J. Mater. Chem. C. 4, 7449-7454.

Farrugia, L. J. (2012). J. Appl. Cryst. 45, 849-854.

Geiser, U., Beno, M. A., Kini, A. M., Wang, H. H., Schultz, A. J., Gates, B. D., Cariss, C. S., Carlson, K. D. \& Williams, J. M. (1988). Synth. Met. 27, A235-A241.
Guionneau, P., Kepert, C. J., Bravic, G., Chasseau, D., Truter, M. R., Kurmoo, M. \& Day, P. (1997). Synth. Met. 86, 1973-1974.

Hiramatsu, T., Yoshida, Y., Saito, G., Otsuka, A., Yamochi, H., Maesato, M., Shimizu, Y., Ito, H. \& Kishida, H. (2015). J. Mater. Chem. C. 3, 1378-1388.

Ishiguro, T., Yamaji, K. \& Saito, G. (1998). Organic Superconductors, 2nd ed. Heidelberg: Springer.

Kurmoo, M., Graham, A. W., Day, P., Coles, S. J., Hursthouse, M. B., Caulfield, J. L., Singleton, J., Pratt, F. L., Hayes, W., Ducasse, L. \& Guionneau, P. (1995). J. Am. Chem. Soc. 117, 12209-12217.

Macrae, C. F., Edgington, P. R., McCabe, P., Pidcock, E., Shields, G. P., Taylor, R., Towler, M. \& van de Streek, J. (2006). J. Appl. Cryst. 39, 453-457.

Martin, L., Turner, S. S., Day, P., Guionneau, P., Howard, J. A. K., Hibbs, D. E., Light, M. E., Hursthouse, M. B., Uruichi, M. \& Yakushi, K. (2001). Inorg. Chem. 40, 1363-1371.

Prokhorova, T. G., Buravov, L. I., Yagubskii, E. B., Zorina, L. V., Khasanov, S. S., Simonov, S. V., Shibaeva, R. P., Korobenko, A. V. \& Zverev, V. N. (2011). CrystEngComm, 13, 537-545.

Prokhorova, T. G., Zorina, L. V., Simonov, S. V., Zverev, V. N., Canadell, E., Shibaeva, R. P. \& Yagubskii, E. B. (2013). CrystEngComm, 15, 7048-7055.

Schultz, A. J., Beno, M. A., Geiser, U., Wang, H. H., Kini, A. M., Williams, J. M. \& Whangbo, M. H. (1991). J. Solid State Chem. 94, 352-361.

Sheldrick, G. M. (2008). Acta Cryst. A64, 112-122.

Sheldrick, G. M. (2015). Acta Cryst. C71, 3-8.

Urayama, H., Yamochi, H., Saito, G., Sato, S., Kawamoto, A., Tanaka, J., Mori, T., Maruyama, Y. \& Inokuchi, H. (1988). Chem. Lett. 17, 463-466.

Valade, L., de Caro, D., Faulmann, C. \& Jacob, K. (2016). Coord. Chem. Rev. 308, 433-444.

Wang, Y.-F., Wang, L.-Y. \& Ma, L.-F. (2008). Z. Anorg. Allg. Chem. 634, 181-185.

Winter, L. E., Steven, E., Brooks, J. S., Benjamin, S., Park, J.-H., de Caro, D., Faulmann, C., Valade, L., Jacob, K., Chtioui, I., Ballesteros, B. \& Fraxedas, J. (2015). Phys. Rev. B, 91, 035437-1-7. 


\section{supporting information}

Acta Cryst. (2018). E74, 1755-1758 [https://doi.org/10.1107/S2056989018015293]

\section{A new compound in the BEDT-TTF family [BEDT-TTF = bis(ethylenedithio)tetra- thiafulvalene] with a tetrathiocyanatocuprate(II) anion, (BEDT-TTF) $)_{4}\left[\mathrm{Cu}(\mathrm{NCS})_{4}\right]$}

\section{Christophe Faulmann, Benoît Cormary, Lydie Valade, Kane Jacob and Dominique de Caro}

Computing details

Data collection: APEX2 (Bruker, 2012); cell refinement: SAINT (Bruker, 2012); data reduction: SAINT (Bruker, 2012); program(s) used to solve structure: SHELXS97 (Sheldrick, 2008); program(s) used to refine structure: SHELXL2018 (Sheldrick, 2015); molecular graphics: Mercury (Macrae et al., 2006); software used to prepare material for publication:

WinGX (Farrugia, 2012).

Bis[bis(ethylenedithio)tetrathiafulvalenium] tetrathiocyanatocuprate(II) bis[bis(ethylenedithio)tetrathiafulvalene]

Crystal data

$\left(\mathrm{C}_{10} \mathrm{H}_{8} \mathrm{~S}_{8}\right)_{2}\left[\mathrm{Cu}(\mathrm{CNS})_{4}\right] \cdot 2 \mathrm{C}_{10} \mathrm{H}_{8} \mathrm{~S}_{8}$

$M_{r}=1834.43$

Monoclinic, $P 2{ }_{1} / c$

$a=16.9036(17) \AA$

$b=21.004(2) \AA$

$c=9.6205(9) \AA$

$\beta=103.071(3)^{\circ}$

$V=3327.1(6) \AA^{3}$

$Z=2$

\section{Data collection}

Bruker Kappa APEXII Quazar CCD diffractometer

Radiation source: microfocus sealed tube Multilayer optics monochromator phi and $\omega$ scans

Absorption correction: multi-scan

(SADABS; Bruker, 2012)

$T_{\min }=0.652, T_{\max }=0.745$

\section{Refinement}

Refinement on $F^{2}$

Least-squares matrix: full

$R\left[F^{2}>2 \sigma\left(F^{2}\right)\right]=0.032$

$w R\left(F^{2}\right)=0.078$

$S=1.02$

5028 reflections

385 parameters

0 restraints
$F(000)=1858$

$D_{\mathrm{x}}=1.831 \mathrm{Mg} \mathrm{m}^{-3}$

Mo $K \alpha$ radiation, $\lambda=0.71073 \AA$

Cell parameters from 7249 reflections

$\theta=2.4-23.5^{\circ}$

$\mu=1.50 \mathrm{~mm}^{-1}$

$T=100 \mathrm{~K}$

Plate, orange

$0.18 \times 0.16 \times 0.02 \mathrm{~mm}$

48335 measured reflections

5028 independent reflections

3816 reflections with $I>2 \sigma(I)$

$R_{\text {int }}=0.070$

$\theta_{\max }=23.7^{\circ}, \theta_{\min }=2.4^{\circ}$

$h=-19 \rightarrow 19$

$k=-23 \rightarrow 23$

$l=-10 \rightarrow 10$

Hydrogen site location: inferred from neighbouring sites

$\mathrm{H}$-atom parameters constrained

$w=1 /\left[\sigma^{2}\left(F_{\mathrm{o}}^{2}\right)+(0.0295 P)^{2}+4.1709 P\right]$

where $P=\left(F_{\mathrm{o}}{ }^{2}+2 F_{\mathrm{c}}{ }^{2}\right) / 3$

$(\Delta / \sigma)_{\max }=0.001$

$\Delta \rho_{\max }=0.38 \mathrm{e} \AA^{-3}$

$\Delta \rho_{\min }=-0.39$ e $\AA^{-3}$ 


\section{Special details}

Geometry. All esds (except the esd in the dihedral angle between two 1.s. planes) are estimated using the full covariance matrix. The cell esds are taken into account individually in the estimation of esds in distances, angles and torsion angles; correlations between esds in cell parameters are only used when they are defined by crystal symmetry. An approximate (isotropic) treatment of cell esds is used for estimating esds involving l.s. planes.

Fractional atomic coordinates and isotropic or equivalent isotropic displacement parameters $\left(\AA^{2}\right)$

\begin{tabular}{|c|c|c|c|c|}
\hline & $x$ & $y$ & $z$ & $U_{\text {iso }} * / U_{\text {eq }}$ \\
\hline $\mathrm{C} 1$ & $-0.0171(2)$ & $0.42002(18)$ & $0.7252(4)$ & $0.0213(9)$ \\
\hline $\mathrm{C} 2$ & $0.0246(2)$ & $0.6302(2)$ & $0.8878(4)$ & $0.0253(10)$ \\
\hline $\mathrm{C} 3$ & $0.8700(2)$ & $0.54753(16)$ & $0.5992(4)$ & $0.0187(9)$ \\
\hline $\mathrm{H} 3 \mathrm{~A}$ & 0.928155 & 0.545997 & 0.647921 & $0.022^{*}$ \\
\hline $\mathrm{H} 3 \mathrm{~B}$ & 0.862454 & 0.520423 & 0.512981 & $0.022^{*}$ \\
\hline $\mathrm{C} 4$ & $0.8478(2)$ & $0.61543(17)$ & $0.5535(4)$ & $0.0209(9)$ \\
\hline $\mathrm{H} 4 \mathrm{~A}$ & 0.890091 & 0.632876 & 0.507824 & $0.025^{*}$ \\
\hline $\mathrm{H} 4 \mathrm{~B}$ & 0.847851 & 0.641325 & 0.639476 & $0.025^{*}$ \\
\hline C5 & $0.7131(2)$ & $0.54291(16)$ & $0.6393(4)$ & $0.0149(8)$ \\
\hline C6 & $0.6896(2)$ & $0.58496(16)$ & $0.5308(4)$ & $0.0160(8)$ \\
\hline $\mathrm{C} 7$ & $0.5595(2)$ & $0.55932(15)$ & $0.6157(4)$ & $0.0149(8)$ \\
\hline $\mathrm{C} 8$ & $0.4827(2)$ & $0.55887(16)$ & $0.6416(4)$ & $0.0154(8)$ \\
\hline C9 & $0.3595(2)$ & $0.53724(16)$ & $0.7503(4)$ & $0.0154(8)$ \\
\hline $\mathrm{C} 10$ & $0.3323(2)$ & $0.57822(16)$ & $0.6408(4)$ & $0.0160(8)$ \\
\hline C11 & $0.2046(2)$ & $0.52444(17)$ & $0.7908(4)$ & $0.0209(9)$ \\
\hline $\mathrm{H} 11 \mathrm{~A}$ & 0.184487 & 0.494450 & 0.711275 & $0.025^{*}$ \\
\hline H11B & 0.171329 & 0.518267 & 0.862179 & $0.025^{*}$ \\
\hline $\mathrm{C} 12$ & $0.1924(2)$ & $0.59208(17)$ & $0.7337(4)$ & $0.0209(9)$ \\
\hline $\mathrm{H} 12 \mathrm{~A}$ & 0.217762 & 0.621861 & 0.810709 & $0.025^{*}$ \\
\hline H12B & 0.133437 & 0.601329 & 0.708352 & $0.025^{*}$ \\
\hline $\mathrm{C} 13$ & $0.8509(2)$ & $0.64170(17)$ & $0.0556(4)$ & $0.0215(9)$ \\
\hline $\mathrm{H} 13 \mathrm{~A}$ & 0.858052 & 0.616445 & 0.144492 & $0.026^{*}$ \\
\hline H13B & 0.892041 & 0.626958 & 0.004150 & $0.026^{*}$ \\
\hline C14 & $0.8670(2)$ & $0.71118(17)$ & $0.0950(4)$ & $0.0203(9)$ \\
\hline H14A & 0.852389 & 0.737338 & 0.007359 & $0.024 *$ \\
\hline H14B & 0.925749 & 0.716993 & 0.136028 & $0.024 *$ \\
\hline $\mathrm{C} 15$ & $0.6912(2)$ & $0.66642(16)$ & $0.0439(4)$ & $0.0132(8)$ \\
\hline $\mathrm{C} 16$ & $0.7133(2)$ & $0.71147(16)$ & $0.1441(4)$ & $0.0156(8)$ \\
\hline C17 & $0.5605(2)$ & $0.69326(16)$ & $0.1286(4)$ & $0.0169(9)$ \\
\hline $\mathrm{C} 18$ & $0.4857(2)$ & $0.69108(16)$ & $0.1551(4)$ & $0.0165(9)$ \\
\hline C19 & $0.3385(2)$ & $0.65929(16)$ & $0.1588(4)$ & $0.0141(8)$ \\
\hline $\mathrm{C} 20$ & $0.3569(2)$ & $0.70712(16)$ & $0.2528(4)$ & $0.0149(8)$ \\
\hline $\mathrm{C} 21$ & $0.1796(2)$ & $0.65689(16)$ & $0.2003(4)$ & $0.0168(9)$ \\
\hline $\mathrm{H} 21 \mathrm{~A}$ & 0.123433 & 0.652063 & 0.142740 & $0.020^{*}$ \\
\hline $\mathrm{H} 21 \mathrm{~B}$ & 0.182206 & 0.635883 & 0.293364 & $0.020^{*}$ \\
\hline $\mathrm{C} 22$ & $0.1970(2)$ & $0.72687(17)$ & $0.2261(4)$ & $0.0229(9)$ \\
\hline $\mathrm{H} 22 \mathrm{~A}$ & 0.152515 & 0.746340 & 0.263158 & $0.028^{*}$ \\
\hline $\mathrm{H} 22 \mathrm{~B}$ & 0.197940 & 0.747725 & 0.134226 & $0.028^{*}$ \\
\hline S1 & $-0.02733(6)$ & $0.37420(5)$ & $0.58614(11)$ & $0.0285(3)$ \\
\hline
\end{tabular}




$\begin{array}{lllll}\text { S2 } & 0.02204(7) & 0.70695(5) & 0.86453(12) & 0.0331(3) \\ \text { S3 } & 0.81075(6) & 0.51479(4) & 0.71701(10) & 0.0169(2) \\ \text { S4 } & 0.75024(6) & 0.62342(4) & 0.43121(10) & 0.0210(2) \\ \text { S5 } & 0.63665(5) & 0.51340(4) & 0.71623(10) & 0.0158(2) \\ \text { S6 } & 0.58730(6) & 0.60578(4) & 0.48575(10) & 0.0173(2) \\ \text { S7 } & 0.46026(6) & 0.51311(4) & 0.77698(10) & 0.0178(2) \\ \text { S8 } & 0.40302(6) & 0.60313(4) & 0.54464(10) & 0.0167(2) \\ \text { S9 } & 0.30860(6) & 0.50474(4) & 0.87210(10) & 0.0198(2) \\ \text { S10 } & 0.23390(6) & 0.60719(4) & 0.57979(10) & 0.0198(2) \\ \text { S11 } & 0.75135(6) & 0.62589(4) & -0.05352(10) & 0.0183(2) \\ \text { S12 } & 0.81090(6) & 0.73927(4) & 0.22123(10) & 0.0178(2) \\ \text { S13 } & 0.58828(6) & 0.64487(4) & -0.00163(10) & 0.0180(2) \\ \text { S14 } & 0.63616(6) & 0.74608(4) & 0.21522(10) & 0.0183(2) \\ \text { S15 } & 0.41224(6) & 0.63676(4) & 0.06567(10) & 0.0180(2) \\ \text { S16 } & 0.45292(6) & 0.74247(4) & 0.27426(11) & 0.0212(2) \\ \text { S17 } & 0.24819(6) & 0.61583(4) & 0.10988(11) & 0.0208(2) \\ \text { S18 } & 0.29242(6) & 0.74148(5) & 0.35111(10) & 0.0232(2) \\ \text { Cu1 } & 0.000000 & 0.500000 & 1.000000 & 0.02233(18) \\ \text { N1 } & -0.0100(2) & 0.45238(15) & 0.8251(4) & 0.0282(8) \\ \text { N2 } & 0.0279(2) & 0.57616(18) & 0.9071(4) & 0.0379(10)\end{array}$

Atomic displacement parameters $\left(\AA^{2}\right)$

\begin{tabular}{lllllll}
\hline & $U^{11}$ & $U^{22}$ & $U^{33}$ & $U^{12}$ & $U^{13}$ & $U^{23}$ \\
\hline C1 & $0.015(2)$ & $0.017(2)$ & $0.030(2)$ & $0.0038(17)$ & $0.0001(19)$ & $0.0093(19)$ \\
C2 & $0.019(2)$ & $0.037(3)$ & $0.021(2)$ & $-0.0072(19)$ & $0.0067(19)$ & $-0.005(2)$ \\
C3 & $0.019(2)$ & $0.017(2)$ & $0.021(2)$ & $0.0019(16)$ & $0.0063(18)$ & $0.0027(16)$ \\
C4 & $0.016(2)$ & $0.016(2)$ & $0.030(2)$ & $-0.0010(16)$ & $0.0057(18)$ & $0.0024(17)$ \\
C5 & $0.019(2)$ & $0.0108(19)$ & $0.016(2)$ & $0.0001(16)$ & $0.0054(17)$ & $-0.0015(16)$ \\
C6 & $0.018(2)$ & $0.0103(19)$ & $0.020(2)$ & $-0.0008(16)$ & $0.0063(17)$ & $-0.0007(16)$ \\
C7 & $0.020(2)$ & $0.0085(18)$ & $0.014(2)$ & $0.0001(16)$ & $0.0009(17)$ & $-0.0022(15)$ \\
C8 & $0.021(2)$ & $0.0120(19)$ & $0.0123(19)$ & $0.0009(16)$ & $0.0007(17)$ & $0.0001(15)$ \\
C9 & $0.018(2)$ & $0.0137(19)$ & $0.015(2)$ & $-0.0001(16)$ & $0.0038(17)$ & $-0.0028(16)$ \\
C10 & $0.022(2)$ & $0.0123(19)$ & $0.014(2)$ & $0.0002(16)$ & $0.0058(17)$ & $-0.0027(16)$ \\
C11 & $0.018(2)$ & $0.024(2)$ & $0.022(2)$ & $-0.0023(17)$ & $0.0077(18)$ & $0.0015(17)$ \\
C12 & $0.022(2)$ & $0.019(2)$ & $0.023(2)$ & $0.0047(17)$ & $0.0083(18)$ & $0.0018(17)$ \\
C13 & $0.016(2)$ & $0.023(2)$ & $0.026(2)$ & $0.0008(17)$ & $0.0075(18)$ & $-0.0040(18)$ \\
C14 & $0.017(2)$ & $0.024(2)$ & $0.021(2)$ & $-0.0037(17)$ & $0.0063(18)$ & $-0.0055(17)$ \\
C15 & $0.014(2)$ & $0.0102(19)$ & $0.017(2)$ & $0.0009(15)$ & $0.0056(16)$ & $0.0041(16)$ \\
C16 & $0.016(2)$ & $0.016(2)$ & $0.016(2)$ & $-0.0024(16)$ & $0.0046(17)$ & $0.0025(16)$ \\
C17 & $0.017(2)$ & $0.0100(19)$ & $0.021(2)$ & $0.0014(16)$ & $-0.0003(18)$ & $-0.0036(16)$ \\
C18 & $0.018(2)$ & $0.0079(19)$ & $0.022(2)$ & $-0.0009(16)$ & $0.0029(18)$ & $-0.0031(16)$ \\
C19 & $0.014(2)$ & $0.0114(19)$ & $0.016(2)$ & $0.0010(15)$ & $0.0025(17)$ & $0.0051(16)$ \\
C20 & $0.018(2)$ & $0.0107(19)$ & $0.015(2)$ & $-0.0001(15)$ & $0.0014(17)$ & $0.0026(15)$ \\
C21 & $0.013(2)$ & $0.020(2)$ & $0.019(2)$ & $-0.0009(16)$ & $0.0042(17)$ & $-0.0043(16)$ \\
C22 & $0.019(2)$ & $0.019(2)$ & $0.031(2)$ & $-0.0010(17)$ & $0.0078(19)$ & $-0.0043(18)$ \\
S1 & $0.0236(6)$ & $0.0350(6)$ & $0.0274(6)$ & $0.0033(5)$ & $0.0066(5)$ & $-0.0062(5)$ \\
S2 & $0.0253(6)$ & $0.0306(6)$ & $0.0430(7)$ & $0.0026(5)$ & $0.0070(5)$ & $0.0175(5)$ \\
& & & & &
\end{tabular}




$\begin{array}{lllllll} & & & & & \\ \text { S3 } & 0.0160(5) & 0.0153(5) & 0.0196(5) & 0.0019(4) & 0.0045(4) & 0.0046(4) \\ \text { S4 } & 0.0236(6) & 0.0184(5) & 0.0222(6) & 0.0021(4) & 0.0079(5) & 0.0087(4) \\ \text { S5 } & 0.0155(5) & 0.0143(5) & 0.0170(5) & 0.0004(4) & 0.0027(4) & 0.0033(4) \\ \text { S6 } & 0.0195(6) & 0.0141(5) & 0.0183(5) & 0.0024(4) & 0.0041(4) & 0.0033(4) \\ \text { S7 } & 0.0188(5) & 0.0169(5) & 0.0175(5) & 0.0019(4) & 0.0037(4) & 0.0032(4) \\ \text { S8 } & 0.0173(5) & 0.0146(5) & 0.0180(5) & 0.0018(4) & 0.0034(4) & 0.0031(4) \\ \text { S9 } & 0.0217(6) & 0.0186(5) & 0.0199(5) & 0.0014(4) & 0.0065(4) & 0.0051(4) \\ \text { S10 } & 0.0189(6) & 0.0222(5) & 0.0185(5) & 0.0052(4) & 0.0046(4) & 0.0044(4) \\ \text { S11 } & 0.0172(5) & 0.0180(5) & 0.0209(5) & -0.0026(4) & 0.0067(4) & -0.0048(4) \\ \text { S12 } & 0.0160(5) & 0.0194(5) & 0.0180(5) & -0.0045(4) & 0.0041(4) & -0.0033(4) \\ \text { S13 } & 0.0139(5) & 0.0148(5) & 0.0243(6) & -0.0003(4) & 0.0024(4) & -0.0042(4) \\ \text { S14 } & 0.0151(5) & 0.0135(5) & 0.0259(5) & -0.0006(4) & 0.0038(4) & -0.0049(4) \\ \text { S15 } & 0.0142(5) & 0.0156(5) & 0.0242(5) & -0.0017(4) & 0.0045(4) & -0.0062(4) \\ \text { S16 } & 0.0163(5) & 0.0179(5) & 0.0300(6) & -0.0027(4) & 0.0066(5) & -0.0093(4) \\ \text { S17 } & 0.0200(6) & 0.0175(5) & 0.0272(6) & -0.0061(4) & 0.0099(5) & -0.0074(4) \\ \text { S18 } & 0.0221(6) & 0.0239(5) & 0.0260(6) & -0.0060(4) & 0.0102(5) & -0.0116(4) \\ \text { Cu1 } & 0.0214(4) & 0.0135(3) & 0.0321(4) & -0.0006(3) & 0.0061(3) & -0.0034(3) \\ \text { N1 } & 0.033(2) & 0.0178(18) & 0.032(2) & 0.0032(16) & 0.0023(17) & 0.0016(17) \\ \text { N2 } & 0.053(3) & 0.026(2) & 0.041(2) & -0.0139(19) & 0.024(2) & -0.0099(18) \\ & & & & & \end{array}$

Geometric parameters $\left(\AA,{ }^{\circ}\right)$

\begin{tabular}{llll}
\hline $\mathrm{C} 1-\mathrm{N} 1$ & $1.161(5)$ & $\mathrm{C} 14-\mathrm{H} 14 \mathrm{~A}$ & 0.9900 \\
$\mathrm{C} 1-\mathrm{S} 1$ & $1.625(4)$ & $\mathrm{C} 14-\mathrm{H} 14 \mathrm{~B}$ & 0.9900 \\
$\mathrm{C} 2-\mathrm{N} 2$ & $1.149(5)$ & $\mathrm{C} 15-\mathrm{C} 16$ & $1.342(5)$ \\
$\mathrm{C} 2-\mathrm{S} 2$ & $1.627(5)$ & $\mathrm{C} 15-\mathrm{S} 11$ & $1.752(4)$ \\
$\mathrm{C} 3-\mathrm{C} 4$ & $1.514(5)$ & $\mathrm{C} 15-\mathrm{S} 13$ & $1.755(4)$ \\
$\mathrm{C} 3-\mathrm{S} 3$ & $1.809(4)$ & $\mathrm{C} 16-\mathrm{S} 12$ & $1.749(4)$ \\
$\mathrm{C} 3-\mathrm{H} 3 \mathrm{~A}$ & 0.9900 & $\mathrm{C} 16-\mathrm{S} 14$ & $1.761(4)$ \\
$\mathrm{C} 3-\mathrm{H} 3 \mathrm{~B}$ & 0.9900 & $\mathrm{C} 17-\mathrm{C} 18$ & $1.346(5)$ \\
$\mathrm{C} 4-\mathrm{S} 4$ & $1.804(4)$ & $\mathrm{C} 17-\mathrm{S} 14$ & $1.755(4)$ \\
$\mathrm{C} 4-\mathrm{H} 4 \mathrm{~A}$ & 0.9900 & $\mathrm{C} 17-\mathrm{S} 13$ & $1.758(4)$ \\
$\mathrm{C} 4-\mathrm{H} 4 \mathrm{~B}$ & 0.9900 & $\mathrm{C} 18-\mathrm{S} 16$ & $1.753(4)$ \\
$\mathrm{C} 5-\mathrm{C} 6$ & $1.357(5)$ & $\mathrm{C} 18-\mathrm{S} 15$ & $1.761(4)$ \\
$\mathrm{C} 5-\mathrm{S} 5$ & $1.742(4)$ & $\mathrm{C} 19-\mathrm{C} 20$ & $1.340(5)$ \\
$\mathrm{C} 5-\mathrm{S} 3$ & $1.753(4)$ & $\mathrm{C} 19-\mathrm{S} 17$ & $1.749(4)$ \\
$\mathrm{C} 6-\mathrm{S} 6$ & $1.740(4)$ & $\mathrm{C} 19-\mathrm{S} 15$ & $1.757(4)$ \\
$\mathrm{C} 6-\mathrm{S} 4$ & $1.751(4)$ & $\mathrm{C} 20-\mathrm{S} 18$ & $1.752(4)$ \\
$\mathrm{C} 7-\mathrm{C} 8$ & $1.377(5)$ & $\mathrm{C} 20-\mathrm{S} 16$ & $1.755(4)$ \\
$\mathrm{C} 7-\mathrm{S} 5$ & $1.731(4)$ & $\mathrm{C} 21-\mathrm{C} 22$ & $1.508(5)$ \\
$\mathrm{C} 7-\mathrm{S} 6$ & $1.732(4)$ & $\mathrm{C} 21-\mathrm{S} 17$ & $1.817(4)$ \\
$\mathrm{C} 8-\mathrm{S} 8$ & $1.726(4)$ & $\mathrm{C} 21-\mathrm{H} 21 \mathrm{~A}$ & 0.9900 \\
$\mathrm{C} 8-\mathrm{S} 7$ & $1.728(4)$ & $\mathrm{C} 21-\mathrm{H} 21 \mathrm{~B}$ & 0.9900 \\
$\mathrm{C} 9-\mathrm{C} 10$ & $1.358(5)$ & $\mathrm{C} 22-\mathrm{S} 18$ & $1.807(4)$ \\
$\mathrm{C} 9-\mathrm{S} 7$ & $1.740(4)$ & $\mathrm{C} 22-\mathrm{H} 22 \mathrm{~A}$ & 0.9900 \\
$\mathrm{C} 9-\mathrm{S} 9$ & $1.742(4)$ & $\mathrm{C} 22-\mathrm{H} 22 \mathrm{~B}$ & 0.9900 \\
$\mathrm{C} 10-\mathrm{S} 10$ & $1.743(4)$ & $\mathrm{S} 3-\mathrm{S} 17^{\mathrm{i}}$ & $3.4702(13)$ \\
$\mathrm{C} 10-\mathrm{S} 8$ & $1.749(4)$ & $\mathrm{S} 3-\mathrm{S} 11^{\mathrm{ii}}$ & $3.5113(13)$ \\
& & &
\end{tabular}




\begin{tabular}{|c|c|c|c|}
\hline $\mathrm{C} 11-\mathrm{C} 12$ & $1.520(5)$ & $\mathrm{S} 4-\mathrm{S} 12$ & $3.4627(13)$ \\
\hline $\mathrm{C} 11-\mathrm{S} 9$ & $1.803(4)$ & $\mathrm{S} 4-\mathrm{S} 14$ & $3.5862(13)$ \\
\hline $\mathrm{C} 11-\mathrm{H} 11 \mathrm{~A}$ & 0.9900 & $\mathrm{~S} 5-\mathrm{S} 11^{\mathrm{ii}}$ & $3.5079(13)$ \\
\hline $\mathrm{C} 11-\mathrm{H} 11 \mathrm{~B}$ & 0.9900 & $\mathrm{~S} 5-\mathrm{S} 17^{\mathrm{i}}$ & $3.5330(13)$ \\
\hline $\mathrm{C} 12-\mathrm{S} 10$ & $1.805(4)$ & $\mathrm{S} 6-\mathrm{S} 7^{\mathrm{i}}$ & $3.5146(13)$ \\
\hline $\mathrm{C} 12-\mathrm{H} 12 \mathrm{~A}$ & 0.9900 & $\mathrm{~S} 9-\mathrm{S} 11^{\mathrm{i}}$ & $3.5216(13)$ \\
\hline $\mathrm{C} 12-\mathrm{H} 12 \mathrm{~B}$ & 0.9900 & $\mathrm{~S} 9-\mathrm{S} 15^{\mathrm{ii}}$ & $3.5707(13)$ \\
\hline $\mathrm{C} 13-\mathrm{C} 14$ & $1.517(5)$ & $\mathrm{S} 9-\mathrm{S} 17^{\mathrm{ii}}$ & $3.5743(14)$ \\
\hline $\mathrm{C} 13-\mathrm{S} 11$ & $1.802(4)$ & $\mathrm{Cu} 1-\mathrm{N} 1$ & $1.932(4)$ \\
\hline $\mathrm{C} 13-\mathrm{H} 13 \mathrm{~A}$ & 0.9900 & $\mathrm{Cu} 1-\mathrm{N} 1^{\mathrm{iii}}$ & $1.932(4)$ \\
\hline $\mathrm{C} 13-\mathrm{H} 13 \mathrm{~B}$ & 0.9900 & $\mathrm{Cu} 1-\mathrm{N} 2^{\mathrm{iii}}$ & $1.942(4)$ \\
\hline $\mathrm{C} 14-\mathrm{S} 12$ & $1.800(4)$ & $\mathrm{Cu} 1-\mathrm{N} 2$ & $1.942(4)$ \\
\hline $\mathrm{N} 1-\mathrm{C} 1-\mathrm{S} 1$ & $179.5(4)$ & $\mathrm{C} 19-\mathrm{C} 20-\mathrm{S} 18$ & $126.5(3)$ \\
\hline $\mathrm{N} 2-\mathrm{C} 2-\mathrm{S} 2$ & $178.3(4)$ & $\mathrm{C} 19-\mathrm{C} 20-\mathrm{S} 16$ & $117.6(3)$ \\
\hline $\mathrm{C} 4-\mathrm{C} 3-\mathrm{S} 3$ & $113.8(3)$ & $\mathrm{S} 18-\mathrm{C} 20-\mathrm{S} 16$ & $115.7(2)$ \\
\hline $\mathrm{C} 4-\mathrm{C} 3-\mathrm{H} 3 \mathrm{~A}$ & 108.8 & $\mathrm{C} 22-\mathrm{C} 21-\mathrm{S} 17$ & $114.8(3)$ \\
\hline $\mathrm{S} 3-\mathrm{C} 3-\mathrm{H} 3 \mathrm{~A}$ & 108.8 & $\mathrm{C} 22-\mathrm{C} 21-\mathrm{H} 21 \mathrm{~A}$ & 108.6 \\
\hline $\mathrm{C} 4-\mathrm{C} 3-\mathrm{H} 3 \mathrm{~B}$ & 108.8 & $\mathrm{~S} 17-\mathrm{C} 21-\mathrm{H} 21 \mathrm{~A}$ & 108.6 \\
\hline $\mathrm{S} 3-\mathrm{C} 3-\mathrm{H} 3 \mathrm{~B}$ & 108.8 & $\mathrm{C} 22-\mathrm{C} 21-\mathrm{H} 21 \mathrm{~B}$ & 108.6 \\
\hline $\mathrm{H} 3 \mathrm{~A}-\mathrm{C} 3-\mathrm{H} 3 \mathrm{~B}$ & 107.7 & $\mathrm{~S} 17-\mathrm{C} 21-\mathrm{H} 21 \mathrm{~B}$ & 108.6 \\
\hline $\mathrm{C} 3-\mathrm{C} 4-\mathrm{S} 4$ & $114.0(3)$ & $\mathrm{H} 21 \mathrm{~A}-\mathrm{C} 21-\mathrm{H} 21 \mathrm{~B}$ & 107.5 \\
\hline $\mathrm{C} 3-\mathrm{C} 4-\mathrm{H} 4 \mathrm{~A}$ & 108.8 & $\mathrm{C} 21-\mathrm{C} 22-\mathrm{S} 18$ & $112.7(3)$ \\
\hline $\mathrm{S} 4-\mathrm{C} 4-\mathrm{H} 4 \mathrm{~A}$ & 108.8 & $\mathrm{C} 21-\mathrm{C} 22-\mathrm{H} 22 \mathrm{~A}$ & 109.0 \\
\hline $\mathrm{C} 3-\mathrm{C} 4-\mathrm{H} 4 \mathrm{~B}$ & 108.8 & $\mathrm{~S} 18-\mathrm{C} 22-\mathrm{H} 22 \mathrm{~A}$ & 109.0 \\
\hline $\mathrm{S} 4-\mathrm{C} 4-\mathrm{H} 4 \mathrm{~B}$ & 108.8 & $\mathrm{C} 21-\mathrm{C} 22-\mathrm{H} 22 \mathrm{~B}$ & 109.0 \\
\hline $\mathrm{H} 4 \mathrm{~A}-\mathrm{C} 4-\mathrm{H} 4 \mathrm{~B}$ & 107.7 & $\mathrm{~S} 18-\mathrm{C} 22-\mathrm{H} 22 \mathrm{~B}$ & 109.0 \\
\hline $\mathrm{C} 6-\mathrm{C} 5-\mathrm{S} 5$ & $116.2(3)$ & $\mathrm{H} 22 \mathrm{~A}-\mathrm{C} 22-\mathrm{H} 22 \mathrm{~B}$ & 107.8 \\
\hline $\mathrm{C} 6-\mathrm{C} 5-\mathrm{S} 3$ & $129.2(3)$ & $\mathrm{C} 5-\mathrm{S} 3-\mathrm{C} 3$ & $101.87(17)$ \\
\hline $\mathrm{S} 5-\mathrm{C} 5-\mathrm{S} 3$ & $114.62(19)$ & $\mathrm{C} 5-\mathrm{S} 3-\mathrm{S} 17^{\mathrm{i}}$ & $97.25(12)$ \\
\hline $\mathrm{C} 5-\mathrm{C} 6-\mathrm{S} 6$ & $117.2(3)$ & $\mathrm{C} 3-\mathrm{S} 3-\mathrm{S} 17^{\mathrm{i}}$ & $149.40(12)$ \\
\hline $\mathrm{C} 5-\mathrm{C} 6-\mathrm{S} 4$ & $128.0(3)$ & $\mathrm{C} 5-\mathrm{S} 3-\mathrm{S} 11^{\mathrm{ii}}$ & $70.65(12)$ \\
\hline S6-C6-S4 & $114.8(2)$ & $\mathrm{C} 3-\mathrm{S} 3-\mathrm{S} 11^{\mathrm{ii}}$ & $114.76(12)$ \\
\hline $\mathrm{C} 8-\mathrm{C} 7-\mathrm{S} 5$ & $121.2(3)$ & $\mathrm{S} 17^{\mathrm{i}}-\mathrm{S} 3-\mathrm{S} 11^{\mathrm{ii}}$ & $94.00(3)$ \\
\hline $\mathrm{C} 8-\mathrm{C} 7-\mathrm{S} 6$ & $123.7(3)$ & $\mathrm{C} 6-\mathrm{S} 4-\mathrm{C} 4$ & $99.35(18)$ \\
\hline $\mathrm{S} 5-\mathrm{C} 7-\mathrm{S} 6$ & $115.1(2)$ & $\mathrm{C} 6-\mathrm{S} 4-\mathrm{S} 12$ & $158.54(12)$ \\
\hline $\mathrm{C} 7-\mathrm{C} 8-\mathrm{S} 8$ & $123.5(3)$ & $\mathrm{C} 4-\mathrm{S} 4-\mathrm{S} 12$ & $95.63(13)$ \\
\hline $\mathrm{C} 7-\mathrm{C} 8-\mathrm{S} 7$ & $121.1(3)$ & $\mathrm{C} 6-\mathrm{S} 4-\mathrm{S} 14$ & $110.11(12)$ \\
\hline $\mathrm{S} 8-\mathrm{C} 8-\mathrm{S} 7$ & $115.4(2)$ & $\mathrm{C} 4-\mathrm{S} 4-\mathrm{S} 14$ & $137.49(12)$ \\
\hline $\mathrm{C} 10-\mathrm{C} 9-\mathrm{S} 7$ & $116.6(3)$ & $\mathrm{S} 12-\mathrm{S} 4-\mathrm{S} 14$ & $49.34(3)$ \\
\hline $\mathrm{C} 10-\mathrm{C} 9-\mathrm{S} 9$ & $129.6(3)$ & $\mathrm{C} 7-\mathrm{S} 5-\mathrm{C} 5$ & $95.85(17)$ \\
\hline S7-C9-S9 & $113.8(2)$ & $\mathrm{C} 7-\mathrm{S} 5-\mathrm{S} 11^{\mathrm{ii}}$ & $102.50(12)$ \\
\hline $\mathrm{C} 9-\mathrm{C} 10-\mathrm{S} 10$ & $127.8(3)$ & $\mathrm{C} 5-\mathrm{S} 5-\mathrm{S} 11^{\mathrm{ii}}$ & $70.81(12)$ \\
\hline $\mathrm{C} 9-\mathrm{C} 10-\mathrm{S} 8$ & $116.6(3)$ & $\mathrm{C} 7-\mathrm{S} 5-\mathrm{S} 17^{\mathrm{i}}$ & $163.25(12)$ \\
\hline $\mathrm{S} 10-\mathrm{C} 10-\mathrm{S} 8$ & $115.6(2)$ & $\mathrm{C} 5-\mathrm{S} 5-\mathrm{S} 17^{\mathrm{i}}$ & $95.28(12)$ \\
\hline $\mathrm{C} 12-\mathrm{C} 11-\mathrm{S} 9$ & $114.3(3)$ & $\mathrm{S} 11^{\mathrm{ii}}-\mathrm{S} 5-\mathrm{S} 17^{\mathrm{i}}$ & 92.97 (3) \\
\hline $\mathrm{C} 12-\mathrm{C} 11-\mathrm{H} 11 \mathrm{~A}$ & 108.7 & $\mathrm{C} 7-\mathrm{S} 6-\mathrm{C} 6$ & $95.43(17)$ \\
\hline $\mathrm{S} 9-\mathrm{C} 11-\mathrm{H} 11 \mathrm{~A}$ & 108.7 & $\mathrm{C} 7-\mathrm{S} 6-\mathrm{S} 7^{\mathrm{i}}$ & $93.63(12)$ \\
\hline
\end{tabular}




\begin{tabular}{|c|c|c|c|}
\hline $\mathrm{C} 12-\mathrm{C} 11-\mathrm{H} 11 \mathrm{~B}$ & 108.7 & $\mathrm{C} 6-\mathrm{S} 6-\mathrm{S} 7^{\mathrm{i}}$ & $93.06(12)$ \\
\hline S9-C11-H11B & 108.7 & $\mathrm{C} 8-\mathrm{S} 7-\mathrm{C} 9$ & $95.80(17)$ \\
\hline $\mathrm{H} 11 \mathrm{~A}-\mathrm{C} 11-\mathrm{H} 11 \mathrm{~B}$ & 107.6 & $\mathrm{C} 8-\mathrm{S} 8-\mathrm{C} 10$ & $95.53(17)$ \\
\hline $\mathrm{C} 11-\mathrm{C} 12-\mathrm{S} 10$ & $114.5(3)$ & $\mathrm{C} 8-\mathrm{S} 8-\mathrm{S} 5^{\mathrm{i}}$ & $90.59(12)$ \\
\hline $\mathrm{C} 11-\mathrm{C} 12-\mathrm{H} 12 \mathrm{~A}$ & 108.6 & $\mathrm{C} 10-\mathrm{S} 8-\mathrm{S} 5^{\mathrm{i}}$ & $97.38(12)$ \\
\hline $\mathrm{S} 10-\mathrm{C} 12-\mathrm{H} 12 \mathrm{~A}$ & 108.6 & $\mathrm{C} 9-\mathrm{S} 9-\mathrm{C} 11$ & $101.52(17)$ \\
\hline $\mathrm{C} 11-\mathrm{C} 12-\mathrm{H} 12 \mathrm{~B}$ & 108.6 & $\mathrm{C} 9-\mathrm{S} 9-\mathrm{S} 11^{\mathrm{i}}$ & $151.62(12)$ \\
\hline $\mathrm{S} 10-\mathrm{C} 12-\mathrm{H} 12 \mathrm{~B}$ & 108.6 & $\mathrm{C} 11-\mathrm{S} 9-\mathrm{S} 11^{\mathrm{i}}$ & $91.85(12)$ \\
\hline $\mathrm{H} 12 \mathrm{~A}-\mathrm{C} 12-\mathrm{H} 12 \mathrm{~B}$ & 107.6 & $\mathrm{C} 9-\mathrm{S} 9-\mathrm{S} 15^{\mathrm{ii}}$ & $77.75(12)$ \\
\hline $\mathrm{C} 14-\mathrm{C} 13-\mathrm{S} 11$ & $114.4(3)$ & $\mathrm{C} 11-\mathrm{S} 9-\mathrm{S} 15^{\mathrm{ii}}$ & $111.30(13)$ \\
\hline $\mathrm{C} 14-\mathrm{C} 13-\mathrm{H} 13 \mathrm{~A}$ & 108.6 & $\mathrm{~S} 11^{\mathrm{i}}-\mathrm{S} 9-\mathrm{S} 15^{\mathrm{ii}}$ & $120.58(3)$ \\
\hline $\mathrm{S} 11-\mathrm{C} 13-\mathrm{H} 13 \mathrm{~A}$ & 108.6 & $\mathrm{C} 9-\mathrm{S} 9-\mathrm{S} 17^{\mathrm{ii}}$ & $115.51(12)$ \\
\hline $\mathrm{C} 14-\mathrm{C} 13-\mathrm{H} 13 \mathrm{~B}$ & 108.6 & $\mathrm{C} 11-\mathrm{S} 9-\mathrm{S} 17^{\mathrm{ii}}$ & $74.71(13)$ \\
\hline $\mathrm{S} 11-\mathrm{C} 13-\mathrm{H} 13 \mathrm{~B}$ & 108.6 & $\mathrm{~S} 11^{\mathrm{i}}-\mathrm{S} 9-\mathrm{S} 17^{\mathrm{ii}}$ & $92.03(3)$ \\
\hline $\mathrm{H} 13 \mathrm{~A}-\mathrm{C} 13-\mathrm{H} 13 \mathrm{~B}$ & 107.6 & $\mathrm{~S} 15^{\mathrm{ii}}-\mathrm{S} 9-\mathrm{S} 17^{\mathrm{ii}}$ & $48.46(3)$ \\
\hline $\mathrm{C} 13-\mathrm{C} 14-\mathrm{S} 12$ & $113.1(3)$ & $\mathrm{C} 10-\mathrm{S} 10-\mathrm{C} 12$ & $100.41(17)$ \\
\hline $\mathrm{C} 13-\mathrm{C} 14-\mathrm{H} 14 \mathrm{~A}$ & 109.0 & $\mathrm{C} 15-\mathrm{S} 11-\mathrm{C} 13$ & $100.20(17)$ \\
\hline $\mathrm{S} 12-\mathrm{C} 14-\mathrm{H} 14 \mathrm{~A}$ & 109.0 & $\mathrm{C} 16-\mathrm{S} 12-\mathrm{C} 14$ & $101.22(17)$ \\
\hline $\mathrm{C} 13-\mathrm{C} 14-\mathrm{H} 14 \mathrm{~B}$ & 109.0 & $\mathrm{C} 16-\mathrm{S} 12-\mathrm{S} 4$ & $68.74(12)$ \\
\hline $\mathrm{S} 12-\mathrm{C} 14-\mathrm{H} 14 \mathrm{~B}$ & 109.0 & $\mathrm{C} 14-\mathrm{S} 12-\mathrm{S} 4$ & $115.70(13)$ \\
\hline $\mathrm{H} 14 \mathrm{~A}-\mathrm{C} 14-\mathrm{H} 14 \mathrm{~B}$ & 107.8 & $\mathrm{C} 15-\mathrm{S} 13-\mathrm{C} 17$ & $94.73(17)$ \\
\hline $\mathrm{C} 16-\mathrm{C} 15-\mathrm{S} 11$ & $128.8(3)$ & $\mathrm{C} 17-\mathrm{S} 14-\mathrm{C} 16$ & $94.53(17)$ \\
\hline $\mathrm{C} 16-\mathrm{C} 15-\mathrm{S} 13$ & $117.4(3)$ & $\mathrm{C} 17-\mathrm{S} 14-\mathrm{S} 4$ & $93.43(12)$ \\
\hline $\mathrm{S} 11-\mathrm{C} 15-\mathrm{S} 13$ & $113.79(19)$ & $\mathrm{C} 16-\mathrm{S} 14-\mathrm{S} 4$ & $65.17(11)$ \\
\hline $\mathrm{C} 15-\mathrm{C} 16-\mathrm{S} 12$ & 128.5 & $\mathrm{C} 19-\mathrm{S} 15-\mathrm{C} 18$ & $94.55(17)$ \\
\hline $\mathrm{C} 15-\mathrm{C} 16-\mathrm{S} 14$ & $117.4(3)$ & $\mathrm{C} 18-\mathrm{S} 16-\mathrm{C} 20$ & $94.76(17)$ \\
\hline $\mathrm{S} 12-\mathrm{C} 16-\mathrm{S} 14$ & $114.1(2)$ & $\mathrm{C} 19-\mathrm{S} 17-\mathrm{C} 21$ & $103.65(17)$ \\
\hline $\mathrm{C} 18-\mathrm{C} 17-\mathrm{S} 14$ & $123.1(3)$ & $\mathrm{C} 20-\mathrm{S} 18-\mathrm{C} 22$ & $98.12(17)$ \\
\hline $\mathrm{C} 18-\mathrm{C} 17-\mathrm{S} 13$ & $122.0(3)$ & $\mathrm{N} 1-\mathrm{Cu} 1-\mathrm{N} 1^{\mathrm{iii}}$ & 180.0 \\
\hline $\mathrm{S} 14-\mathrm{C} 17-\mathrm{S} 13$ & $114.9(2)$ & $\mathrm{N} 1-\mathrm{Cu} 1-\mathrm{N} 2^{\mathrm{iii}}$ & $89.53(14)$ \\
\hline $\mathrm{C} 17-\mathrm{C} 18-\mathrm{S} 16$ & $123.5(3)$ & $\mathrm{N} 1^{\mathrm{iii}}-\mathrm{Cu} 1-\mathrm{N} 2^{\mathrm{iii}}$ & $90.47(14)$ \\
\hline $\mathrm{C} 17-\mathrm{C} 18-\mathrm{S} 15$ & $121.2(3)$ & $\mathrm{N} 1-\mathrm{Cu} 1-\mathrm{N} 2$ & $90.46(14)$ \\
\hline $\mathrm{S} 16-\mathrm{C} 18-\mathrm{S} 15$ & $115.2(2)$ & $\mathrm{N} 1^{\mathrm{iii}}-\mathrm{Cu} 1-\mathrm{N} 2$ & $89.53(14)$ \\
\hline $\mathrm{C} 20-\mathrm{C} 19-\mathrm{S} 17$ & $128.9(3)$ & $\mathrm{N} 2{ }^{\mathrm{iii}}-\mathrm{Cu} 1-\mathrm{N} 2$ & 180.0 \\
\hline $\mathrm{C} 20-\mathrm{C} 19-\mathrm{S} 15$ & $117.6(3)$ & $\mathrm{C} 1-\mathrm{N} 1-\mathrm{Cu} 1$ & $175.2(3)$ \\
\hline $\mathrm{S} 17-\mathrm{C} 19-\mathrm{S} 15$ & $113.5(2)$ & $\mathrm{C} 2-\mathrm{N} 2-\mathrm{Cu} 1$ & $151.6(4)$ \\
\hline
\end{tabular}

Symmetry codes: (i) $-x+1,-y+1,-z+1$; (ii) $x, y, z+1$; (iii) $-x,-y+1,-z+2$. 